\title{
Lattice-based structures for studying percolation in two- dimensional grain networks
}

\author{
Brent L. Adams \\ b_I_adams@byu.edu \\ John A. Basinger \\ David T. Fullwood \\ dfullwood@byu.edu
}

Follow this and additional works at: https://scholarsarchive.byu.edu/facpub

Part of the Mechanical Engineering Commons

Original Publication Citation

Acta Materialia 54 (26) 1381-1388

\section{BYU ScholarsArchive Citation}

Adams, Brent L.; Basinger, John A.; and Fullwood, David T., "Lattice-based structures for studying percolation in two-dimensional grain networks" (2006). Faculty Publications. 326.

https://scholarsarchive.byu.edu/facpub/326

This Peer-Reviewed Article is brought to you for free and open access by BYU ScholarsArchive. It has been accepted for inclusion in Faculty Publications by an authorized administrator of BYU ScholarsArchive. For more information, please contact ellen_amatangelo@byu.edu. 


\title{
Lattice-based structures for studying percolation in 2-D grain networks
}

\author{
David T. Fullwood, John A. Basinger, Brent L. Adams \\ Department of Mechanical Engineering, Brigham Young University, \\ Provo, UT 84604, USA.
}

Tel: 801-422-2059 email: dfullwood@,byu.edu

Journal article available at www.actamat-journals.com

\section{Keywords:}

grain boundaries, grain boundary structure, percolation.

\begin{abstract}
The applicability of standard lattice percolation models to a random 2-D grain structure is explored. A random network based on the triangle lattice is proposed as a more appropriate model, and results in a higher percolation threshold ( 0.711 compared with 0.653 for the standard hexagonal lattice). The triple junction constraint inherent in grain boundary structures is subsequently applied to the new network. This results in a lowering of the percolation threshold to 0.686 ; this is opposite to its effect on the standard hexagonal lattice. The effect of varying the network's 'grain shape' distribution on the percolation threshold is also considered.
\end{abstract}

\section{Introduction}

Percolation theory is a natural mathematical tool for studying certain properties of grains boundary networks. In particular, the distinct properties of high angle random boundaries (HAR) when compared against other grain boundaries (low angle random - LAR - and / 
or coincident site lattice - CSL) creates a natural definition of open and closed bonds that may be used in the percolation model. This in turn may be used to predict path-based phenomena such as fracture $([1],[2],[3])$ or corrosion $([4],[5])$.

In the case of fracture, grain boundaries consist of regions where atomic interfaces are less ordered, and result in a higher potential energy. If a fracture surface develops along the grain boundary, this potential energy will reduce the amount of energy required to form the new surface, and may result in intergranular fracture becoming the dominant mode of failure.

In a brittle material, the crack driving force, $\mathrm{G}$, across a grain is $2 \gamma_{\mathrm{s}}$, as usual. The value of $\mathrm{G}$ along a grain boundary will be approximately $2\left(1.2 * \gamma_{\mathrm{s}}\right)-\gamma_{\mathrm{b}}$, where the value of 1.2 represents a higher surface-generation energy for new surface along a grain boundary [6], and $\gamma_{b}$ is the extra potential energy at the grain boundary due to disorder. The value of $\gamma_{b}$ is often approximated to be zero for LAR and CSL boundaries, and assigned a single value for all HAR boundaries (e.g. [7]). More exact values can be calculated [8], but the two-value approximation is often sufficient.

Various methods have been used to investigate intergranular fracture using the two value estimation for $\gamma_{b}$, including semi-empirical [7], minimal surface [9-10] and fuse-lattice [11]. However, the division of boundaries into high or low energies (open or closed bonds) allows percolation theory to be used as yet another tool in this area of investigation. 
Traditionally percolation theory as applied to grain boundaries has utilised the hexagonal (or 'honeycomb') lattice as the basis for the theory (e.g., [12],[13],[14]). This lattice naturally reflects the triple junction network found in a grain structure.

A recent paper [15] reported results from studying percolation effects across real grain boundaries in a 2-D section of 304 stainless steel, and compared them with the traditional models. The data was taken from a detailed OIM scan with a $6 \mu \mathrm{m}$ step size over a $4 \mathrm{~mm}$ square sample of the material. HAR grain boundaries were defined to be the 'open bonds'. The HAR boundaries were those with misorientation above $15^{\circ}$ that did not fall under the criterion for a CSL boundary. This criterion was varied until a cluster of HAR boundaries spanned the sample. A figure of the percolating network is shown in Fig. 1.

Non-contributing loops were removed from the analysis, and a bond was defined as a segment of grain boundary between two triple junctions.

Percolation was found to occur at $\mathrm{P}(\mathrm{HAR})=0.46$ (i.e. the probability that a given boundary is HAR was 0.46 ). This compares with a theoretical value of 0.653 for the standard hexagonal lattice [16], or 0.659 for the standard hexagonal lattice with constraints at the triple junctions [17] (using the value obtained for 'twinned' material).

As discussed in [15], the main reason for the discrepancy appears to be the presence of two distinct networks within the grain structure - a 'contributing' network of HAR and 
non-HAR grain boundaries that determine the main characteristics of the HAR clusters; and within this network, a 'non-contributing' network of CSL and LAR boundaries that lie within the outer network, and bias the ratio of non-HAR boundaries. These belong to twins and sub-grains, and constitute up to around $66 \%$ of the total grain boundary length, or $45 \%$ of the total number of 'bonds' (segments of grain boundaries between triple junctions).

However, while this explanation gives a plausible reason for the discrepancy between the measured and the theoretical percolation threshold, the question remained open as to the applicability of the standard lattice model to random grain structures.

If we define an edge in the grain boundary network to be a segment connecting two adjacent triple junctions, then it may be readily seen from Fig. 1 that the number of edges per grain is somewhat random, and is certainly not accurately described by the hexagonal lattice, which has a constant six edges per grain.

This paper studies the effect of such differences when it comes to determining the percolation threshold. A more suitable model derived from lattice structures is proposed and compared with the random grain network. The percolation threshold for the new structure is determined. The affect of applying a triple junction constraint, to better reflect actual grain properties, is also investigated. 
In a preprint brought to the authors' attention during peer review of this paper, a limited study of an 'irregular lattice' is undertaken in the context of scaling laws [18]. This structure is obtained starting from the traditional hexagonal lattice and switching boundaries to vary the number of edges for random grains. While the methodology and scope of the preprint is significantly different from this paper, it is of interest that irregular structures have recently been of interest to major authors in this field.

\section{Description of the new structure}

The basis of the following approach is to assume that a 2-D graph of grain boundaries has only triple junctions. This is consistent with the standard approaches to grain boundary networks [12]-[14]. Secondly, lengths of the grain boundary sections are ignored, and each section between grain boundary junctions is considered as a single bond (again consistent with the standard percolation approach). Thus grain boundary networks will be compared with lattice based structures in a topological manner.

Based upon these assumptions, the starting point is to define a random 'pseudo lattice' that has similar structure to a 2-D grain boundary network. We start from a standard triangular lattice as shown in Fig. 2. Each vertex is assigned 3 bonds (as shown) to form the standard unit cell for the lattice. Thus there are 3 bonds per vertex (in this paper the number of bonds per vertex means the number of bonds divided by the number of vertices - not the number of bonds meeting at each vertex). Since we require a triple

junction at each vertex we must delete an average of 1.5 bonds per vertex. 
Unit cells are populated from left to right, and from bottom to top of the lattice. For the current unit cell, if the vertex is already attached to a total of $b$ bonds (from unit cells to the left and below), then (3-b) bonds are randomly chosen from the unit cell to complete the triple junction. For the left and bottom edges of the lattice, the value of $b$ is chosen randomly. A sample resultant pseudo lattice is shown in Fig. 3.

In order to determine whether this is a reasonable representation of a 2-D grain structure, we compare it to a real grain sample. Clearly the number of bonds per vertex will be correct if the real grain structure is made up purely of triple junctions. The other parameter that may be used to compare the topological equivalence of the networks is the distribution of the number of bonds (or edges) per grain. In the sample (Fig. 1, and [15]) the average number of edges per grain is 4.8 over 413 grains; in our example pseudo lattice, the number is 5.6 over 183 grains (later analysis over a large number of grains showed that the number of edges per grain converges to 6 ; for a small number of grains the edge effects - larger grains being more likely to terminate outside the current window - skewed the results). Figure 4 shows the distribution of number of edges per grain for the real and theoretical networks.

It should be noted that the number of edges per grain calculated for the 304 stainless steel was counted by eye, hence very short edges would not necessarily be resolved. This may explain the rather high number of grains with 3 edges amongst the 413 grains. 
Notwithstanding the limited data, the pseudo lattice is clearly much closer to the actual grain structure than a hexagonal lattice where every grain has 6 edges.

Having established that the pseudo lattice is a closer topological fit to a real grain structure than the hexagonal lattice, we expect that a real grain structure will have similar percolation properties to the pseudo lattice.

\section{Discussion of expected results}

Before analysing the percolation properties of the pseudo lattice, we consider how we might expect it to compare to standard lattices. Consider the percolation thresholds for the three most common lattices given in Table 1 (the values are for bond percolation):

Table 1: Percolation thresholds for standard lattices ([11]); note that the number of bonds per vertex is $1 / 2$ the number of bonds meeting at each vertex.

\begin{tabular}{|l|l|l|l|l|}
\hline Lattice & $\begin{array}{l}\text { Number } \\
\text { of bonds } \\
\text { per vertex }\end{array}$ & $\begin{array}{l}1 / \text { (number } \\
\text { of bonds per } \\
\text { vertex) }\end{array}$ & $\begin{array}{l}\text { Percolation } \\
\text { threshold }\end{array}$ & $\begin{array}{l}\text { Average number of open } \\
\text { bonds meeting at each } \\
\text { vertex at percolation }\end{array}$ \\
\hline Triangular & 3 & 0.33 & 0.347 & 2.08 \\
\hline Square & 2 & 0.5 & 0.5 & 2 \\
\hline Hexagonal & 1.5 & 0.67 & 0.653 & 1.96 \\
\hline
\end{tabular}

The percolation threshold is strongly related to the number of bonds per vertex. This seems to be intuitive given that the number of bonds per vertex determines the density of paths in the lattice (consistent with standard texts, a percolation path is assumed to follow open bonds - e.g. [19]). 
Gurland (see for example, [20]) proposed that for 3-D percolation, an average of 1.5 open bonds at each vertex was an approximate critical value independent of whether the graph was random or a regular lattice. In 2-D, it may be seen from Table 1 that the number of open bonds connecting to each vertex at percolation is an average of approximately 2 . This is another way of making the same observation that the percolation threshold is approximately $1 /$ (number of bonds per vertex).

From this point of view, the pseudo lattice (and grain structure) has 1.5 bonds per vertex, and hence is expected to have a similar percolation threshold to the hexagonal lattice. This is consistent with the normal representation of a grain structure as a hexagonal lattice.

One must now question the effect of the random nature of the pseudo lattice when compared to the regular hexagonal lattice, and whether this will significantly change the expected percolation threshold. To aid the discussion we note that both the square and hexagonal lattices can be obtained by deleting 1 or 1.5 bonds per vertex, respectively from the standard triangle lattice (see Fig. 5 and Fig. 6).

Take the case of the square lattice. Deleting one bond per vertex of the triangular lattice removes $1 / 3$ of the bonds; if these had been removed in a random manner, one would expect that $\frac{0.347}{1-1 / 3}=0.52$ of the remaining bonds should be open to arrive at the 
percolation threshold (consistent with the percolation threshold for the triangle lattice of 0.347). In fact the actual fraction requiring removal is 0.5 . Hence the non-random removal of the first $1 / 3$ of the bonds has not had a huge effect on the percolation threshold.

Similarly, for the hexagonal lattice, had we deleted $1 / 2$ of the bonds in a random manner, we would expect that $\frac{0.347}{1-0.5}=0.694$ of the remaining bonds should be open to arrive at the percolation threshold. In this case, the actual value is 0.653 .

In the case of the pseudo lattice, since the bonds are removed in a somewhat random manner (as opposed to the structured removal of bonds to form the honeycomb lattice), one would expect that the percolation threshold will be closer to the value of 0.694 . This was the starting hypothesis of the authors.

\section{Percolation threshold results for the pseudo lattice}

To determine an actual value for the percolation threshold for the pseudo lattice, a number of random lattices were created and the threshold found numerically for each lattice (with a search step size of 0.001). Various methods of deciding the percolation threshold were tried with very similar results. The chosen method was to take the value of $\mathrm{P}(\mathrm{HAR})$ that had an equal weight of percolating values below as the weight of nonpercolating values above it. The weight was simply the distance of the value from the calculated percolation threshold. 
Percolation was registered if a continuous path of HAR boundaries occurred from left to right AND / OR from bottom to top of the window. The size of the window was increased until convergence of the percolation threshold was obtained.

A typical set of results is illustrated in Table 2 for a single pseudo lattice.

Table 2: Typical data from percolation searches on a single pseudo lattice with varying $\mathrm{P}$ (HAR). For this case the calculated threshold is 0.7114 . A 'Percolation' value of $0 / 1$ indicates that percolation has not / has occurred (respectively) for this value of $\mathrm{P}(\mathrm{HAR})$.

\begin{tabular}{|c|l|l|l|l|l|l|l|}
\hline Percolation & P(HAR) & & & & & & \\
\hline 0 & 0.6910 & 0 & 0.7010 & 0 & 0.7110 & 1 & 0.7210 \\
0 & 0.6920 & 0 & 0.7020 & 1 & 0.7120 & 1 & 0.7220 \\
0 & 0.6930 & 0 & 0.7030 & 0 & 0.7130 & 1 & 0.7230 \\
0 & 0.6940 & 1 & 0.7040 & 0 & 0.7140 & 1 & 0.7240 \\
0 & 0.6950 & 0 & 0.7050 & 1 & 0.7150 & 1 & 0.7250 \\
0 & 0.6960 & 1 & 0.7060 & 0 & 0.7160 & 1 & 0.7260 \\
0 & 0.6970 & 0 & 0.7070 & 0 & 0.7170 & 1 & 0.7270 \\
0 & 0.6980 & 0 & 0.7080 & 1 & 0.7180 & 1 & 0.7280 \\
0 & 0.6990 & 0 & 0.7090 & 1 & 0.7190 & 1 & 0.7290 \\
0 & 0.7000 & 1 & 0.7100 & 1 & 0.7200 & 1 & 0.7300 \\
\hline
\end{tabular}

Approximately forty values of $\mathrm{P}(\mathrm{HAR})$ were assessed on more than 10 different randomly generated pseudo lattices of approximately 6000 grains. A number of pseudo lattices of 16000 grains were also used to confirm the accuracy of the smaller models. The results were remarkably consistent with a standard deviation of less than 0.002 $(0.25 \%)$

The average percolation threshold value was 0.711 . This was slightly higher than the expected value of 0.694 (by 2\%). 
To validate the model a standard hexagonal lattice was assessed in exactly the same manner, and a percolation threshold of 0.6513 obtained. This is slightly below the published value of 0.6527 (by $0.2 \%$ ), but generally supports the accuracy of the models and methodology.

\section{Application of the triple junction constraints}

It has been observed (see for example, [14]) that the misorientation values of three grain boundaries meeting at a triple junction are not completely independent. If $\mathbf{M}_{\mathrm{i}}$ is the misorientation matrix for the $\mathrm{i}^{\text {th }}$ grain boundary, then:

$$
\mathbf{M}_{1} \mathbf{M}_{2} \mathbf{M}_{3}=\mathbf{I}
$$

where $\mathbf{I}$ is the identity matrix.

The constraint would generally be applied in a natural manner by assigning random (3D) orientations to the grains, and calculating the resultant (2D) misorientation. For ease of application of the constraint in our case, it is convenient to deal solely with randomly assigned misorientations and applying the constraint derived from (1) as follows [14]:

$$
\theta_{\max } \leq \theta_{\mathrm{i}}+\theta_{\mathrm{j}}
$$

$\theta_{\max }$ is the maximum misorientation of the three boundaries at a given triple junction, and $\theta_{\mathrm{i}}, \theta_{\mathrm{j}}$ are the other two misorientations.

An arbitrary maximum misorientation of $22.5^{\circ}$ was chosen for the exercise, and a misorientation was labelled as HAR if the angle was greater than $15^{\circ}$. This turned out to 
be a convenient combination that resulted in less effort required to satisfy (2). Note that the study in [14] reported that the percolation results on a standard lattice were insensitive to both actual crystal symmetry (and hence maximum misorientation), and defined HAR limit. A target $\mathrm{P}(\mathrm{HAR})$ was chosen, and a given angle randomly selected between $0^{\circ}$ and $15^{\circ}$ (i.e. LAR) or $15^{\circ}$ and $22.5^{\circ}$ (i.e. HAR) based upon this probability. If the resultant boundaries meeting at a given junction did not satisfy (2), the process was repeated. A limit was placed upon the number of attempts at each junction. However, the constraint was violated at less than $0.1 \%$ of the junctions.

The final $\mathrm{P}(\mathrm{HAR})$ that resulted from this algorithm was slightly higher than the target; thus the final value was the one used in the analysis.

To illustrate the effect of the constraint, and by way of validation, we define $\mathrm{J}_{\mathrm{i}}(\mathrm{i}=1,2,3)$ to be the probability that a given junction will have i LAR (i.e. non-HAR) boundaries meeting there. For example, if $\mathrm{P}(\mathrm{HAR})=1$, then $\mathrm{J}_{3}=\mathrm{J}_{2}=\mathrm{J}_{1}=0$ since there are no LAR boundaries, and $\mathrm{J}_{0}=1$. Values of $\mathrm{J}_{\mathrm{i}}$ are plotted in Fig. 7 for the unconstrained case, and for the constrained case.

These figures compare favourably with those in [14], and indicate that the method for choosing the grain boundary orientations is reasonable. To be more specific, the constrained values lie between those for the general and those for the fibre case described in [14], and closer to the general case. We will discuss these cases in more detail after presenting the results of the constrained pseudo lattice model. 
As for the unconstrained case, several hundred percolation searches were undertaken on different pseudo lattices, and with varying P(HAR). Once again the results were extremely consistent (the standard deviation was 0.003 ). The effect of the constraint was to reduce the percolation threshold from 0.711 to 0.686 .

To put this result in context we quote the results from several models on the standard hexagonal model reported in [12]; see Table 3 . The fibre case represents a material with a preferred crystallographic axis (such as for an extruded material); the twinned case represents a material with significant twinning, and where the open / closed bonds are taken to be non-CSL / CSL respectively (i.e. P(HAR) refers to P(non-CSL)).

Table 3: Percolation thresholds $\left(\mathrm{P}_{\mathrm{c}}\right)$ and approximate values of $\left(\mathrm{J}_{0}+\mathrm{J}_{1}\right)$ for various cases.

\begin{tabular}{|l|l|l|}
\hline Model & $\mathrm{P}_{\mathrm{c}}(\mathrm{HAR})$ & $\begin{array}{l}\mathrm{J}_{0}+\mathrm{J}_{1} \text { at } \\
\mathrm{P}(\mathrm{HAR})=0.7\end{array}$ \\
\hline Random hexagonal [16] & 0.653 & 0.21 \\
\hline Fibre constrained hexagonal [14] & 0.601 & 0.33 \\
\hline General constrained hexagonal [14] & 0.676 & 0.29 \\
\hline Twinned constrained hexagonal [17] & 0.659 & 0.27 \\
\hline Random pseudo lattice (this paper) & 0.711 & 0.21 \\
\hline Constrained pseudo lattice (this paper) & 0.686 & 0.29 \\
\hline
\end{tabular}

$\mathrm{J}_{0}$ and $\mathrm{J}_{1}$ are the fraction of junctions with 3 and 2 HAR boundaries respectively. Thus, the value of $\mathrm{J}_{0}+\mathrm{J}_{1}$ gives the fraction of junctions that contribute to HAR paths. If the $\mathrm{J}_{0}$ and $\mathrm{J}_{1}$ junctions were distributed randomly through the material, it would clearly be the case that a higher value of $\mathrm{J}_{0}+\mathrm{J}_{1}$ would mean a higher probability of a percolating path, and therefore a lower value of $\mathrm{P}_{\mathrm{c}}$. However, for all of the constrained models the value of 
$\mathrm{J}_{0}+\mathrm{J}_{1}$ is higher than the respective random case; yet the value of $\mathrm{P}_{\mathrm{c}}$ is not consistently lowered due to the constraint.

It is observed in [14] that the application of the triple junction constraint tends to cluster the HAR bonds. In the case of the fibre texture the clusters tend to be 'strings', thus increasing the probability of a percolation path. Furthermore, in this case the value of $\mathrm{J}_{0}+\mathrm{J}_{1}$ is highest. Both of these facts lead to a lowering of $\mathrm{P}_{\mathrm{c}}$. In the case of the general texture, the clusters of HAR bonds form tighter groups, and actually decrease the probability of percolation, despite the rise in $\mathrm{J}_{0}+\mathrm{J}_{1}$. Hence the nature of the clustering appears to have a larger affect than the change in J values.

In the case of the pseudo lattice, the clustering also results in a 'patchier' network than the random case. However, the clusters appear to help paths circumnavigate the occasional larger grains, rather than resulting in small loops of HAR bonds (see Fig. 8) This, combined with the higher value of $\mathrm{J}_{0}+\mathrm{J}_{1}$, reduces the percolation threshold from the random case.

\section{Effect of Varying the Grain Shape Distribution}

The results presented so far relate to the natural pseudo-lattice created using the method outlined, and which has a particular distribution of grain shapes. Since the object of the paper is to argue that grain shape has a significant effect on percolation thresholds we 
now apply a simple method of changing the grain shape distribution and observe the effect on the threshold.

The method is implemented by increasing (or decreasing) the probability that adjacent bonds be deleted in the formation of the original pseudo lattice, thus increasing (or decreasing) the probability of 'large' grains (grains with a higher number of edges) being created. Two cases were studied - one (Case A) with increased, and the other (Case B) with decreased probabilities of deleting adjacent bonds. The resultant grain shape distributions are plotted in Fig. 9 along with the natural pseudo lattice. The results for the various cases, with and without the triple junction constraint, are given in Table 4.

Table 4: Results for percolation thresholds with various grain shape distributions

\begin{tabular}{|l|l|l|l|l|}
\hline & $\begin{array}{l}\text { Hexagonal } \\
\text { lattice }\end{array}$ & $\begin{array}{l}\text { Natural } \\
\text { pseudo lattice }\end{array}$ & Case A & Case B \\
\hline Mean number of edges & 6 & 6 & 6 & 6 \\
\hline Standard deviation & 0 & 3.68 & 4.83 & 3.07 \\
\hline $\mathrm{P}_{\mathrm{c}}$ - random case & 0.653 & 0.711 & 0.739 & 0.691 \\
\hline $\mathrm{P}_{\mathrm{c}}$ - constrained case & 0.676 & 0.686 & 0.717 & 0.666 \\
\hline
\end{tabular}

As one might expect, increasing the probability of larger grains in Case A makes percolation more difficult, as percolating paths have to circumnavigate these grains; thus the percolation threshold is higher. Similarly, taking the opposite approach (Case B) reduces the percolation threshold. The triple junction constraint has a similar effect in terms of lowering the threshold for each of these cases. The results give some indication of how the percolation threshold will alter with grain shape distribution. In Fig. 10 we plot $\mathrm{P}_{\mathrm{c}}$ vs. standard deviation for the four cases in Table 4. We also plot a best fit quadratic. 


\section{Summary}

The objective of this study was the investigation of percolation on lattice-based networks that represented the topology of real grain structures more closely than the traditional hexagonal lattice.

A random pseudo lattice composed of triple junctions has been obtained from the standard triangle lattice, and is proposed as a closer fit to real 2-D grain structures. The average number of edges in a single real grain structure (0.48) was found to be lower than that for the pseudo lattice (0.56). This is likely to be due to effects such as sub grains (see [15]), but more data is required for a true comparison. However, the distribution of number of edges per grain for the real case and the pseudo lattice is reasonably close.

The percolation threshold for the pseudo lattice has been found to be 0.711 . This is slightly higher than the expected value of 0.694 , and significantly higher than the standard hexagonal lattice value of 0.653 .

For percolation in 2-D, an approximate average value of 2 open bonds meeting at each vertex is required. The resultant threshold of 0.711 is equivalent to 2.13 open bonds at each vertex, which is slightly higher than the value for the triangle lattice. 
We have argued that the percolation threshold would be close to the value of 0.694 . This has proven to be the case. The reason for the slightly higher than expected value is presumably due to the obstacles created by the randomly placed large grains that necessitate longer paths on average to cross the sample; this results in the requirement for a higher percentage of open bonds for percolation.

The triple junction constraint - representing the relationship between misorientation angles at a triple junction for real grain boundaries - has been applied to the pseudo lattice, and results in the lowering of the percolation threshold to 0.686 . This is the opposite of the effect seen on the hexagonal lattice, where the threshold was raised from 0.653 to 0.676 . Thus the final separation of the constrained hexagonal and pseudo lattice thresholds turns out to be quite small.

The effect of varying the grain shape distribution in the pseudo lattice has been demonstrated. The presence of a higher percentage of larger grains raises the percolation threshold, as one might expect. The change in $\mathrm{P}_{\mathrm{c}}$ for the small number of distributions tested seems to correlate to the standard deviation from the mean number of edges per grain. The triple junction constraint consistently lowers the threshold for each variation of the pseudo lattice.

The standard deviation in the numerical results for the calculated percolation thresholds, across a series of randomly constructed pseudo lattices, is very small, and indicates that the results from such structures will be stable. 
Finally, it is noted that this study involves a 2-D construct. This is consistent with the majority of percolation studies, and allows comparison with a wide range of 2-D studies. Furthermore, comparison with actual grain shapes is currently only reasonable for 2-D data (although 3-D data is growing due to techniques such as serial sectioning). However, any percolation phenomenon of interest will generally be occurring in a 3-D environment; hence the above study would ideally be expanded to 3-D. While the authors have not yet attempted to do this, one way might be to begin with the regular cubic lattice. If a line is defined by the intersection of any perpendicular faces, then the lattice has six lines meeting at each vertex. In a similar way to that described for the pseudo lattice, one might randomly delete the lines (and hence the faces that meet there) until only three meet at each vertex. The construct will not be as neat, since deleting some lines will result in deleted vertices, but the overall goal of a random arrangement of cells with only three cells meeting on any given line should be achieved. No doubt there are various alternative (and better) methods of arriving at a similar 3-D construction.

Acknowledgement: This work was supported primarily by the MRSEC program of the National Science Foundation under DMR-0079996.

\section{References}

[1] Gertsman VY, Tangri K. Acta Mater. 1997; 45: 4107.

[2] Wang G, Zuo L, Esling C. Phil. Mag. 2002; A82: 2499. 
[3] Watanabe T. Mater. Sci. Eng. 1994; A176: 39.

[4] Pan Y, Olson T, Adams BL. Can. Metall. Q. 1995; 34: 147.

[5] Palumbo G, King PJ, Aust KT, Erb U, Lichtenberger PC. Scripta Metall. Mater. 1991; 25: 1775 .

[6] Cottrell AH, Materials Science and Technology, Vol 6, 1989 pp 121

[7] Gokhale AM, Deshpande NU, Denzer DK, Liu J, Relationship between fracture toughness, fracture path, and microstructure of 7050 aluminum alloy: Part II. Multiple micromechanisms-based fracture toughness model, Metallurgical and Materials Transactions A, April 1998; Vol 29A, pp 1203

[8] Wolf D, Merkle KL, Correlation between the structure and energy of grain boundaries in metals, in Materials Interfaces: Atomic-Level Structure \& Properties, editors Wolf \& Yip, Chapman \& Hall, 1992

[9] Holm EA, McGovney GN, Minimum surface formation energy for three-dimensional intergranular fracture, Mat. Res. Soc. Symp. Proc., 1999; Vol 539 [10] Raisanen VI, Quasistatic cracks and minimal energy surfaces, Physical review letters, Jan 1998; vol 80 \#2, [11] Srolovitz DJ, Yang WH, Najafabadi R, Wang HY, LeSar R, Microstructural and segregation effects in the fracture of polycrystals, in Materials interfaces, 1992 (editors Wolf and Yip).

[12] Minich RW, Schuh CA, Kumar M, Physical Review B 2002;66:052101.

[13] Frary M, Schuh CA, Applied Physics Letters 2003;83:3755.

[14] Frary M, Schuh CA, Physical Review B 2004;69:134115. 
[15] Basinger JA, Homer ER, Fullwood DT, Adams BL, Two dimensional grain boundary percolation in alloy 304 stainless steel, Scripta Mat, 2005; 53/8: 959-963 [16] Stauffer D, Aharony A. Introduction to Percolation Theory $2^{\text {nd }}$ Ed. Bristol, PA: Taylor and Francis; 1992. p.17.

[17] Frary M, Schuh CA, "Connectivity and percolation behaviour of grain boundary networks in three dimensions", Philosophical Magazine, April 2005; Vol. 85, No. 11: $1123-1143$

[18] Frary M, Schuh CA, Grain Boundary Networks: Scaling Laws, Preferred Cluster Structure, and Their Implications for Grain Boundary Engineering, Submitted to Acta Mat., January 2005

[19] Grimmett G, Percolation, $2^{\text {nd }}$ Edition, Springer, 1999

[20] Janzen J, J Appl Phys 1975;46:966 


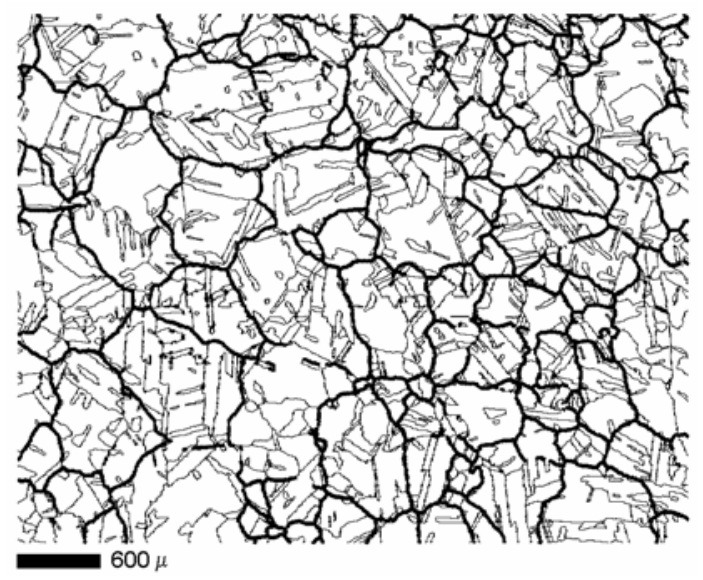

Figure 1: Grain boundary network for 304 stainless steel studied in [15]; the heavier boundaries are HAR, while the light ones are CSL or LAR.

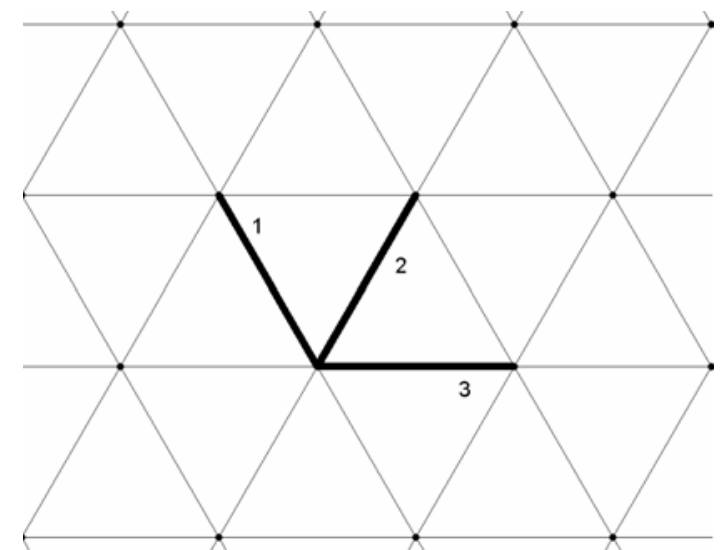

Figure 2: The basis triangle lattice with 3 bonds per vertex

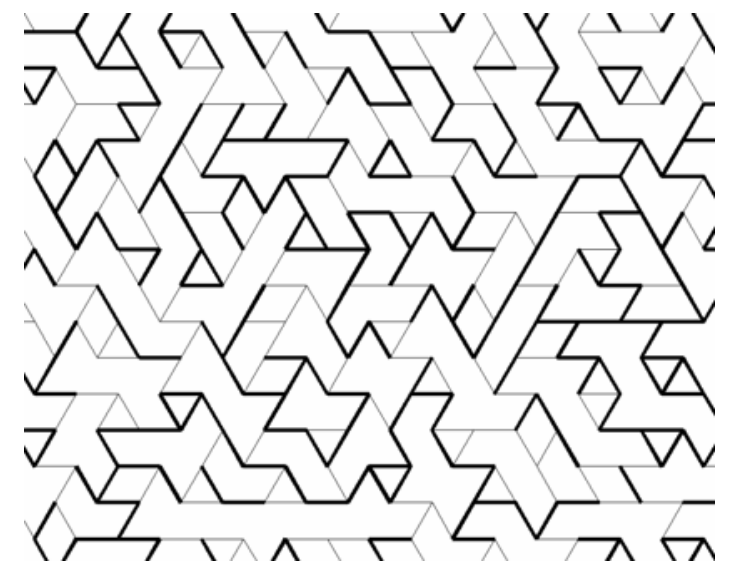

Figure 3: A random pseudo lattice with $\mathrm{P}(\mathrm{HAR})=0.65$; the heavier lines represent the HAR boundaries. 


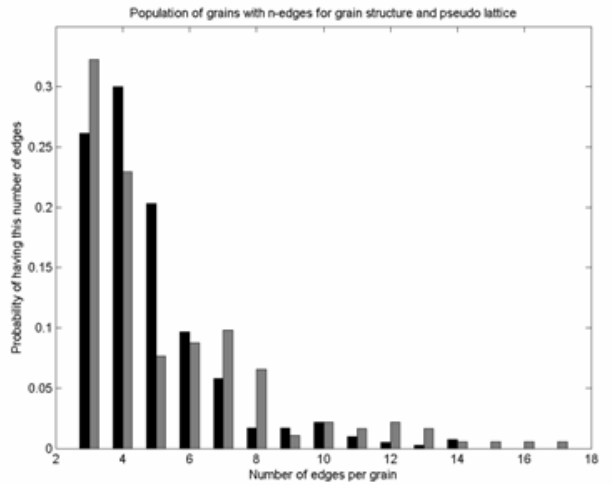

Figure 4: Comparison of number of edges per grain for a real grain structure (black) and the pseudo lattice (grey) introduced in this paper

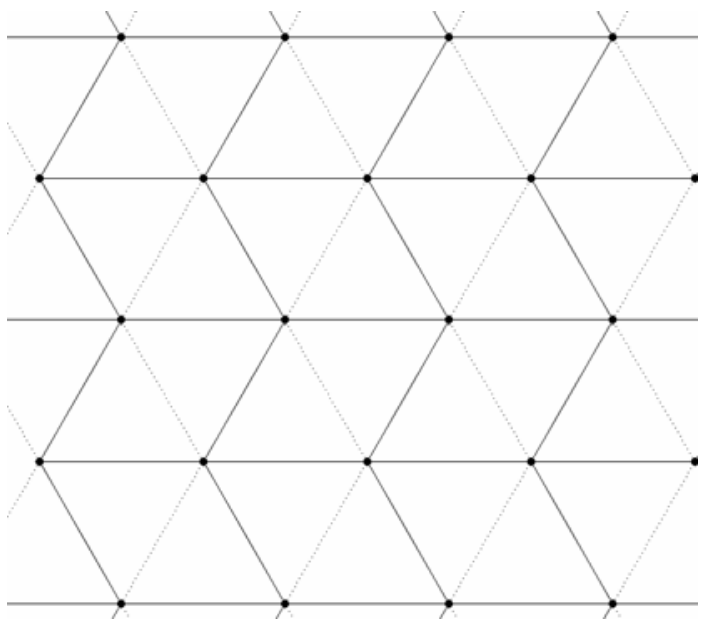

Figure 5: Obtain a square lattice by deleting the dotted lines from the triangle lattice and translating every other horizontal line.

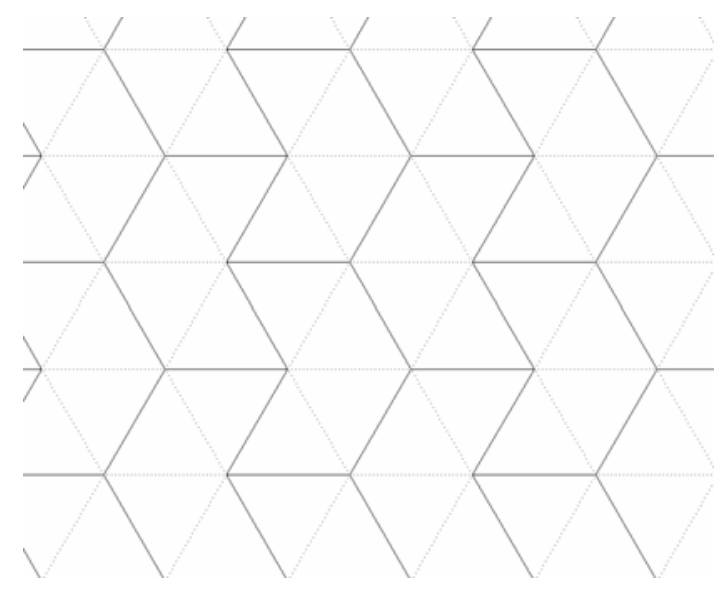


Figure 6: Obtain a hexagonal lattice by deleting the dotted bonds in the triangle lattice, and expanding the gap between the required vertices to arrive at hexagons.
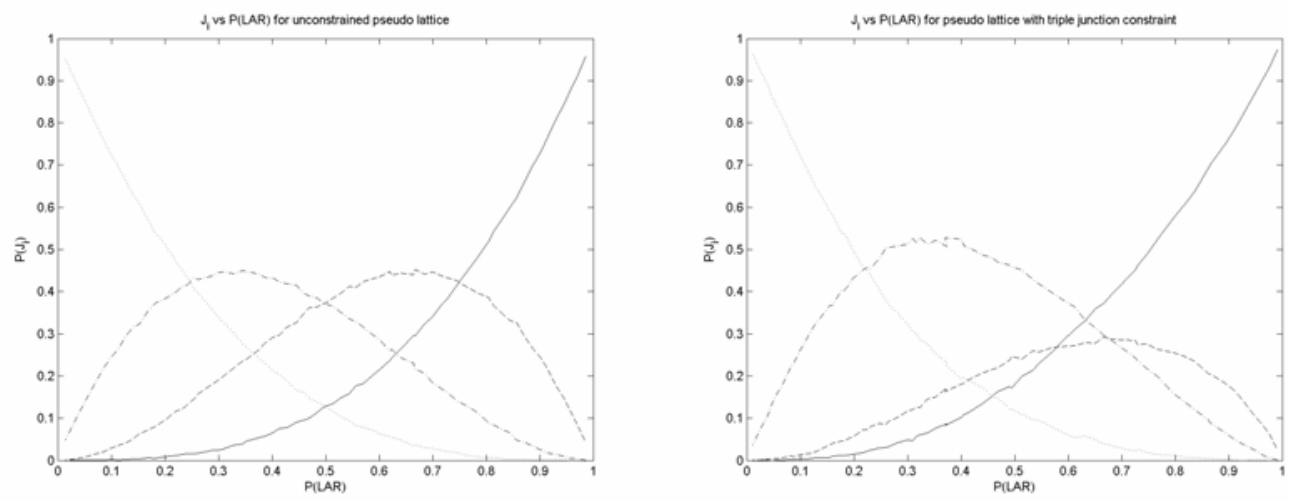

Figure 7: $\mathrm{J}_{\mathrm{i}}$ for the unconstrained (a) and constrained (b) cases on a pseudo lattice. The graphs are for: $\mathrm{J}_{0}-$ dotted; $\mathrm{J}_{1}-$ dot-dash; $\mathrm{J}_{2}-$ dashed; $\mathrm{J}_{3}-$ solid.
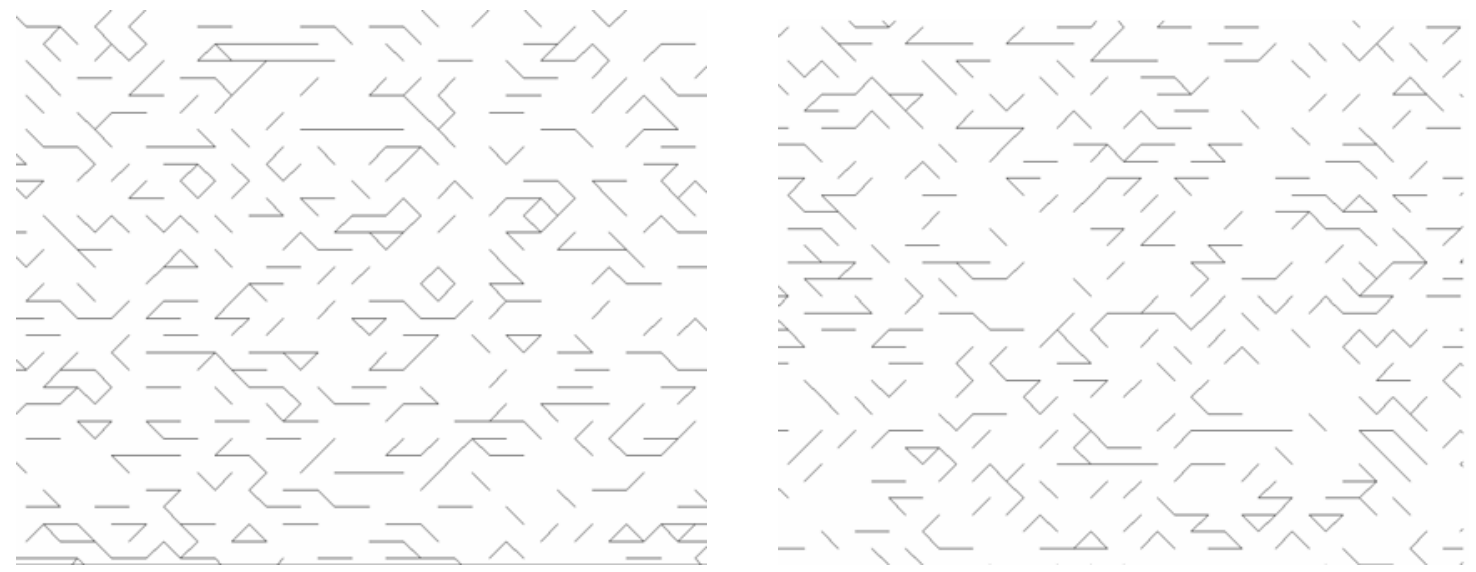

Figure 8: $\mathrm{P}(\mathrm{HAR})=0.4$ for constrained (a) and unconstrained (b) cases respectively. The constraint results in larger HAR clusters / more closed paths. 


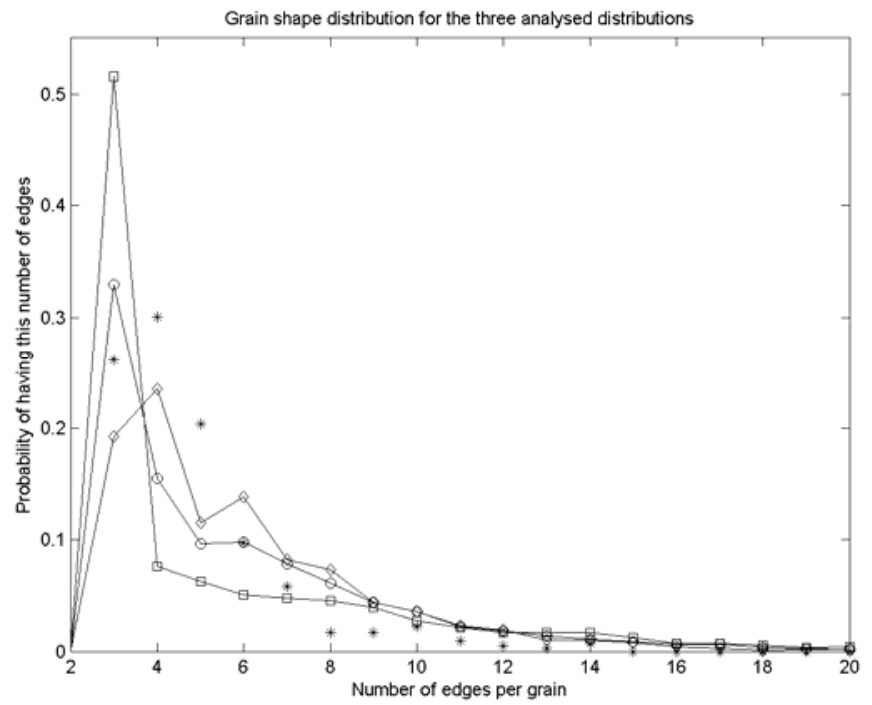

Figure 9: Grain shape distributions for the natural pseudo lattice (circles), Case A (squares) and Case B (diamonds). Data from the real sample (Fig. 4) is also shown (asterisks). Note that the data continues to the right with Case A dominating for high numbers of edges (albeit at low probability).

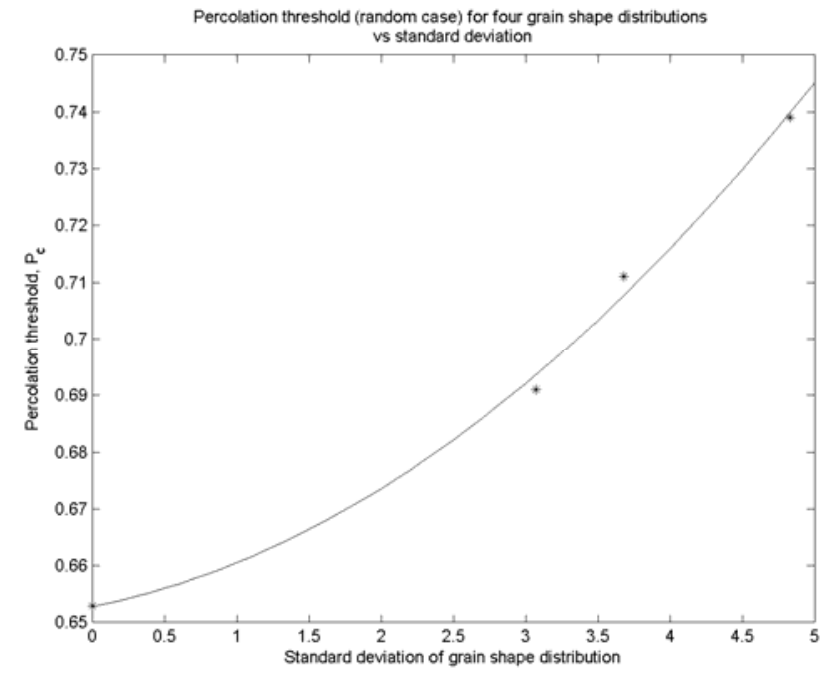

Figure 10: Percolation threshold (for the random case) vs. standard deviation (from the mean of 6 edges per grain) for the four distributions given in Table 4 . A best fit quadratic is also plotted. 